\title{
Low-cost, open source bioelectric signal acquisition system
}

\author{
Enzo Mastinu, Bo Håkansson and Max Ortiz-Catalan
}

\begin{abstract}
Bioelectric potentials provide an intuitive source of control in human-machine interfaces. In this work, a low-cost system for bioelectric signals acquisition and processing was developed and made available as open source. A single module based on the ADS1299 (Texas Instruments, USA) can acquire up to 8 differential or single-ended channels with a resolution of 24 bits and programmable gain up to $24 \mathrm{~V} / \mathrm{V}$. Several modules can be daisy-chained together to increase the number of input channels. Opto-isolated USB communication was included in the design to interface safely with a personal computer. The system was designed to be compatible with a low-cost and widely available microcontroller development platform, namely the Tiva LaunchPad (Texas Instruments, USA) featuring an ARM Cortex-M4 core. We made the source files for the PCB, firmware, and high-level software available online (GitHub: ADS_BP). Digital processing was used for float conversion and filtering. The high-level software for control and acquisition was integrated into an already existent open source platform for advanced myoelectric control, namely BioPatRec. This integration provide a complete system for intuitive myoelectric control where signal processing, machine learning, and control algorithms are used for the prediction of motor volition and control of robotic and virtual devices.
\end{abstract}

\section{INTRODUCTION}

Human-machine interfacing via bioelectric signals has been historically pursue for rehabilitation and research purposes, as well as for entrainment in more recent years. Myoelectric control based on pattern recognition is currently an active field of research which exploits the natural occurrence of bioelectric potential to intuitively control artificial limbs, exoskeletons, or other rehabilitation devices. Although such technology was shown feasible for prosthetics in home-trials since the 1970's [1], it is not until recently that a commercially system is available (Coapt, USA). The dissemination of technologies taking advantages of bioelectric signals is partially limited by the availability of affordable hardware and software. Commercially available bioelectric acquisition systems are costly, bulky, and often restricted to operate using the manufacturer software only. This limits the usability of such hardware in divergent research or mobile applications.

Open source projects have tried to oppose this trend, such as the BCI2000 project [2] for brain computer interfacing (BCI)

Research supported by the Swedish Research Council (Vetenskapsrådet), VINNOVA, and the Promobilia Foundation.

E. Mastinu is with the Biomechatronics and Neurorehabilitation Laboratory at the Dept. of Signals and System, Chalmers University of Technology, Gothenburg, Sweden (e-mail: enzo@chalmers.se).

B. Håkansson is with the Dept. of Signals and System, Chalmers University of Technology, Gothenburg, Sweden (e-mail: boh@chalmers.se).

M. Ortiz-Catalan is with the Biomechatronics and Neurorehabilitation Laboratory at the Dept. of Signals and System, Chalmers University of Technology, and Integrum $\mathrm{AB}$, both in Gothenburg, Sweden (e-mail: maxo@chalmers.se). exclusively dedicated to software, or the ModularEEG [3] and OpenBCI [4] projects which provide open source software and hardware with focus on EEG for the same purpose. EMG has received attention by its own in low-cost systems for single [5] and multi channels [6], [7] acquisition devices. However the related hardware and software is not openly available for further development.

In 2013, a platform for the research and development of advanced control strategies using bioelectric signals was released as open source, namely BioPatRec [8]. This platform provides all the code necessary for an intuitive control of virtual or robotic devices, including signals acquisition and processing, feature extraction and selection, pattern recognition, and control algorithms, as well as a virtual reality environment. However, the lack of affordable hardware for the acquisition of the bioelectric signals has limited several BioPatRec users to offline processing only. In this study, we present an open source solution for a complete low-cost, human-machine interfacing system based in bioelectric signals (EMG/EEG/ECG). This includes acquisition hardware together with the embedded software needed for processing bioelectric signals ( $\geq 8$ channels), along with the interface to high-level software for the prediction of motor volition.

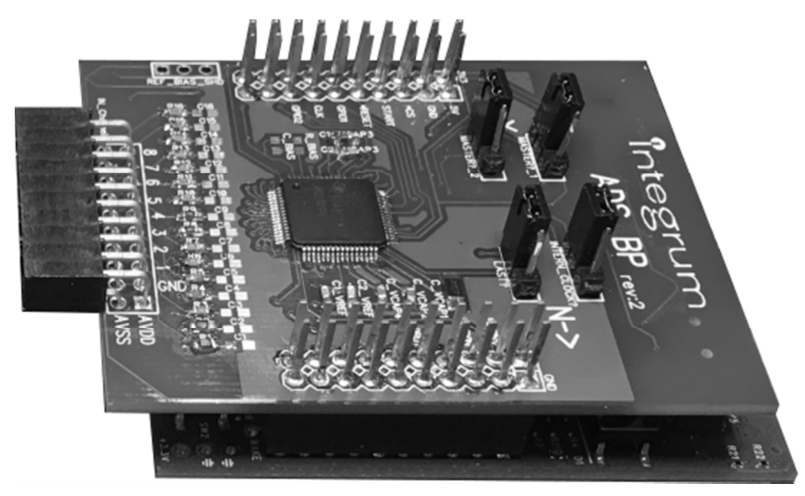

Figure 1. ADS_BP stacked on top of a Tiva LaunchPad.

\section{SYSTEM ARCHITECTURE \& DETAILS}

The acquisition system is divided in three parts: 1) the Analog Front-End (AFE) and optically isolated communication in a dedicated printed circuit board (PCB), 2) the microcontroller unit (MCU) and its embedded software for signal acquisition and processing, and 3) computer software for control and communication with a personal computer (PC). In order to realize a system that is easily and widely available, we selected a low-cost MCU developing platform: the Tiva LaunchPad (Texas Instruments, USA). 
Mastinu, E., Håkansson, B., and Ortiz-Catalan, M., Low-cost, open source bioelectric signal acquisition system, in Proceedings of the 14th Annual International Conference on Wearable and Implantable Body Sensor Networks of the IEEE Engineering in Medicine and Biology Society. Eindhoven, May 9-12, 2017.

The Tiva LaunchPad has a double 20 pin connectors that allows stacking expansion boards on the top and bottom (booster packs), thus allowing access to power lines, multiple GPIOs and peripherals. A PCB was designed containing the $\mathrm{AFE}$ and isolated communication interface which takes advantages of this stackable architecture (Fig. 1), and thus more than one PCB can be connected in the same stack to expand the number of channels. The system design and evaluation performance is described in this work along with a brief functional description of the firmware and the PC software needed for establishing communication and signal acquisition.

\section{A. $A D S B P$}

The ADS1299 (Texas Instruments, USA) was selected as the AFE due to its low cost and previous performance benchmarking with other AFEs [9]. A single ADS1299 provides 8 simultaneous bipolar channels with a resolution of 24 bits, making it suitable for EMG/EEG/ECG applications. Together with its relatively affordable price ( $\sim 50$ USD), one of the major strength of the ADS1299 is the embedded combination of analog and digital components in the same package. This reduces the board space and makes it a versatile AFE where parameters such as output data rate, gain, and reference electrode connection, can be modified via software, thus eliminating the need of exchanging external component. Despite the analog-digital integration, the noise rejection performances remained suitable for the application [9]. The ADS_BP's PCB was fully designed using DipTrace (Novarm, Ukraine), a schematic and PCB development software available also in freeware version; Fig. 2 shows a view of top and bottom layers; PCB size is $52 \times 65 \mathrm{~mm}$.
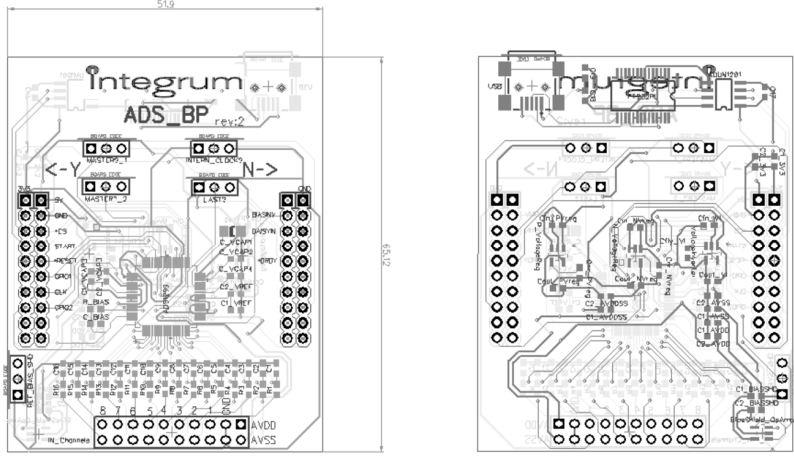

Figure 2. ADS_BP PCB: Top (left) and Bottom (right) layers.

Resistor from R1 to R16 and capacitors from $\mathrm{C} 1$ to $\mathrm{C} 16$ are included as initial conditioning of the signals. The ADS1299 has an inner decimation filter that introduces a pass-band trend that repeats itself to each multiple of the oversampler frequency of $1.048 \mathrm{MHz}$. For this reason an antialiasing input filter is recommended for each input channel; resistors and capacitors were included in the PCB design for this purpose. Alternatively, these components can be used for compensating the absence of an internal DC decoupling capacitor with a RC high-pass filter option. Moreover, if none of this is required, zero Ohm resistors can be placed in
R1 to R16 shorting the electrodes directly to the ADS1299 input terminals. This last option was selected for our testing, and digital IIR filters were applied digitally by the MCU in real-time.

An amplifier for the leads' shielding was included in the board and can be used for improving the noise rejection. Moreover, the ADS1299 offers the possibility to improve CMRR by using a built in amplifier to drive a bias voltage similarly to a Driven-Right-Leg circuit [10]. This option is also available in the ADS_BP via a 3-pole jumper for selecting the electrode connection, and it must be enabled via firmware by modifying the proper configuration registers. Moreover, the ADS_BP is designed to allow multiple stacking of other ADS_BP boards; several jumpers are provided and must be correctly set on all boards to define which board is the master and which are the slaves. When using this configuration it must be kept in mind that the SPI lines are shared and the maximum number of connectable boards will depend on the selected output data rate. For example, when the ADS1299 is operated at a 2-kSPS data rate with a 4-MHz SPI clock, 10 devices can be daisychained. In this situation, the overall current consumption must be also considered; the ADS1299 power requirement is estimated in around $5 \mathrm{~mW}$ per channel.

In addition, an opto-isolated USB port was included in the board to provide a safe user interface $(>25-\mathrm{kV} / \mu \mathrm{s})$. It is based on a FT232RL (FTDI, UK), for converting UART-to-USB, and ADUM1201 (Analog Devices, USA) for isolating the AFE from the power line. All this circuitry can be avoided on the slaves ADS_BP in the case of multiple boards.

\section{B.MCU board}

The MCU board is in charge of acquiring the raw samples from the AFE, process, and then forward them to a PC via the isolated USB connection. The Tiva LaunchPad is based on an ARM Cortex-M4 core which can run up to $80 \mathrm{MHz}$. The internal memories of $256 \mathrm{~KB}$ of Flash and $32 \mathrm{~KB}$ of SRAM, combined with the Floating-Point dedicated unit, allow the programmer to implement bioelectric signal processing algorithms. This MCU is also equipped with two USB connectors, one for programming and real-time debugging, and another one for communication. It must be pointed out that when connecting the device to a user for bioelectric recordings, these two USB ports should not be used as they are not isolated from the mains. The communication with a PC or any other potentially dangerous back-end should be done via the opto-isolated USB in the ADS BP. Data transfer can also be achieved in several other ways. For instance, off-the-shell wireless modules can be placed in a board designed in the same stackable style, giving the system the possibility of wireless communication. Two wireless modules, Bluetooth and WiFi, were tested with the ADS_BP and power consumption was measured.

\section{C.ADS_BP Firmware}

The firmware was written in two versions: in the first version, namely Read\&Send, the MCU only reads the raw 
byte packages and forwards them to the PC. In the second version, the MCU was used for digital signal processing (DSP) applying float conversions and filtering. Both versions of the firmware are provided online (Github: ADS_BP).

1) Firmware: Read\&Send

After powering the device, the MCU enters in idle mode waiting for commands coming on the UART port. An UART dedicated Interrupt Service Routine (ISR) is used to manage the commands decode and execution. When the expected start command is received, the MCU set the continuous acquisition mode on the ADS1299 and data streaming begins. From that moment, another ISR is used to trigger the nDRDY line of the ADS1299 and acquire the bytes package on the SPI port every time new conversion results are available. Channels samples are represented in "complement of two" format and included into a 27 bytes fixed-sized package that the ADS1299 periodically sends out on the SPI lines every time the results of a new conversion are ready. After the start command, the role of the MCU becomes basically to wait for each new falling edge of the nDRDY line, then read the 27 bytes package from the AFE, and finally forward it via the UART port. The data streaming is interrupted via a predefined stop command. In this case, the ADS1299 is set in idle mode and the nDRDY line interrupt disabled. It is worthy to note that this firmware was based on the idea that all the required conversion of data, from an ADC output format to voltage, is done on the PC side. Transmission error control was not implemented in the communication. In addition, a LED output will alert the user if the system is in standby or in acquisition mode by changing the blinking frequency from $2 \mathrm{~Hz}$ to $10 \mathrm{~Hz}$ after the start command is received.

2) Firmware: $D S P$

In this firmware the MCU converts the raw packages of information into a more intelligible format. The three bytes associated to each channel for each sample are firstly converted from "complement of two" into voltage, expressed as float single precision number. This requires a roughly $20 \%$ increase of the required transmission bandwidth respect to the previous firmware version. Digital IIR filters can be enabled and applied to acquired signals sample-by-sample: a first order $50 \mathrm{~Hz}$ notch and a second order $20 \mathrm{~Hz}$ high-pass were implemented. The DSP firmware shares basically all the rest of the code with the Read\&Send version, and therefore it behaves as mentioned above. It is a slave device which by default is in standby waiting for commands to execute.

\section{D.PC Software: BioPatRec}

In order to provide a complete and open source system for bioelectric signals acquisition, efforts were also spent on the high-level software. The device reported in this paper was integrated into an already existing open source project: BioPatRec [11]. BioPatRec represents a research platform developed on Matlab (Mathworks, USA) for acquiring and processing bioelectric signals, as well as for implementing and testing pattern recognition algorithms for human motor control [8]. The necessary routines for BioPatRec to use the acquisition system here proposed is provided in the
BioPatRec release FRA (June 2016). Both firmware versions were integrated in BioPatRec, leaving to the interested developers the decision on where to focus their coding efforts, firmware or high-level software.
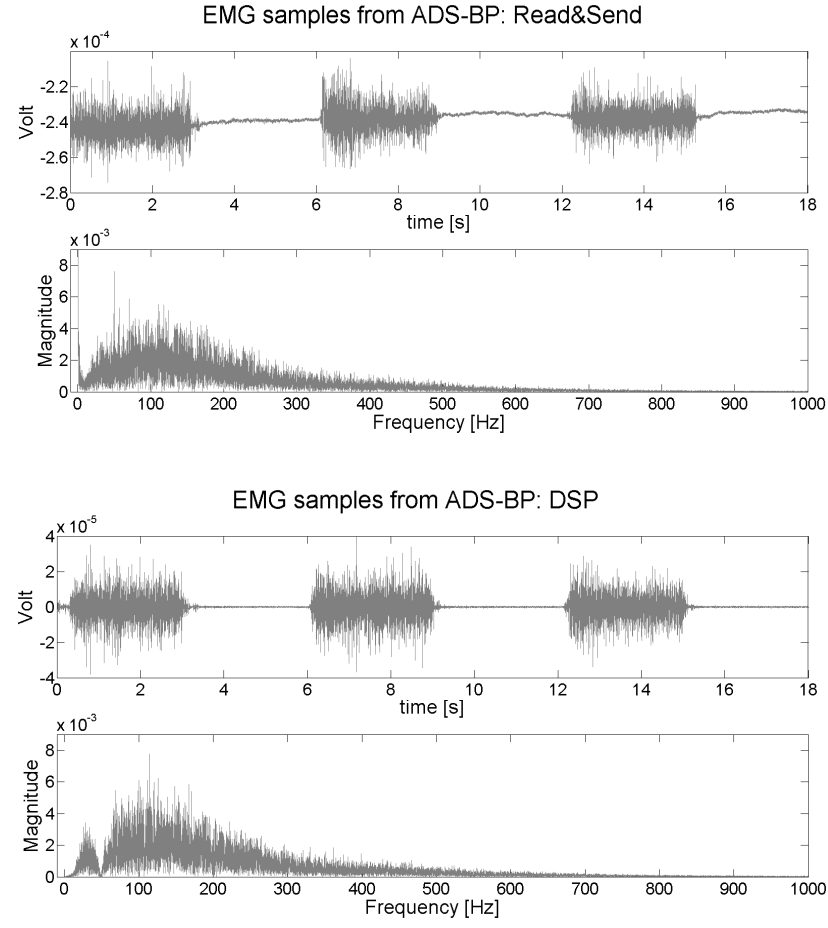

Figure 3. Sample of recordings using BioPatRec and the two firmware versions: Read\&Send (top) and DSP (bottom). The recordings include both time and frequency domains of one EMG channel (extensor digitorum muscle) during open hand movement, three seconds of contraction followed by three seconds of rest, alternated for three times.

\section{TEST \& RESULTS}

The ADS_BP board was assembled and stacked on the top of a Tiva LaunchPad. Communication with a PC was done via the opto-isolated USB port of the ADS_BP. Zero Ohm resistors were placed on position $\mathrm{R} 1$ to $\mathrm{R} 1 \overline{6}$, tiding directly all electrodes to the input terminals of the ADS1299. The CMRR and Input-Referred Noise were measured at $120 \mathrm{~dB}$ and $2.5 \mu \mathrm{V}$, respectively [9]. BioPatRec was used to record EMG signals from the forearm of eight able-bodied subjects. Recording sessions were acquired asking the subject to execute 10 hand and wrist movements with 3 repetitions each, alternating 3 seconds of contraction with 3 seconds of relaxation. The classification accuracy for these movements was $96.2 \pm 2.3$, and overall SNR of $23 \pm 4.3 \mathrm{~dB}$. The details on classification and SNR comparing other AFEs are published elsewhere [9]. Fig. 3 shows an example of signals acquired using the different firmware versions where the absence of the DC component in the DSP version can be observed. 
The cost evaluation of the bio-signals acquisition system (8 channels) described in this work is presented in Table 1 using prices from the last quarter of 2015 .

TABLE I. COST EVALUATION

\begin{tabular}{|l|c|}
\cline { 2 - 2 } \multicolumn{1}{c|}{} & Price [USD] \\
\hline ADS_BP components & 80 \\
\hline Tiva LaunchPad & 15 \\
\hline 8-Channels System Price & 95 \\
\hline
\end{tabular}

Table 2 shows current consumption and battery life of the system with wireless communication, both Bluetooth and WiFi. The WT12 (Bluegiga, USA) and Compact-UART (Wiicom, Italy) modules were used in this experiment. The system was powered by a 0.8 Ah battery and a 5 Volts voltage regulation board based on a TPS62133 (Texas Instruments, USA). The current consumption was measured varying the state of the device from idle to recording. As expected, Bluetooth was found as a less power demanding transmission in comparison to WiFi. On the other hand, WiFi allows for a wider bandwidth. Overall, the battery life was found suitable for portable, battery powered applications.

TABLE II. BATTERY LIFE ESTIMATION

\begin{tabular}{|l|c|c|c|}
\hline $\begin{array}{c}\text { Mode vs } \\
\text { Current [mA] }\end{array}$ & Standby & Acquisition & $\begin{array}{c}\text { Estimated Battery } \\
\text { Life (Acquisition) [h] }\end{array}$ \\
\hline USB & 61 & 65 & 12 \\
\hline WiFi & 195 & 295 & 3 \\
\hline Bluetooth & 69 & 81 & 10 \\
\hline \hline
\end{tabular}

\section{CONCLUSION}

A versatile, portable, and low-cost bioelectric signal acquisition system was developed and made freely available as open source. It includes the PCB design, as well as the $\mathrm{MCU}$ firmware and Matlab routines needed to acquire data using a PC. The firmware is available in two versions: raw data transmission and digital data processing. The use of firmware will depend on the application: Read\&Send is perhaps suggested for developers focused in multi-modular acquisition $(\geq 16$ channels) since the DSP on the MCU introduces a processing delay, proportional to the number of channels, that needs to be matched with an efficient real-time performance. The cost evaluation of this system is below \$100 USD. Functional and power consumption tests revealed its potential for portable bioelectric acquisition. Tests reported affordable power consumption in USB mode, as well as in the experiment of wireless transmission, thus confirming the potential of developing a wearable battery powered device. The PCB design and firmware can be further improved, for instance, refinement of the transmission protocol sacrificing bandwidth by error coding strategies could be used to improve the robustness of the data transfer. The digital filters could be further improved by increasing the order while keeping an acceptable real-time performance. Analogously, the same steps could be implemented in PC software. In addition, power optimization can be introduced using the MCU sleep modes offered by the Cortex-M4 core.
This work ultimate aim is to facilitate the initiation of programmers or biomedical engineers into the fields of biosignals acquisition, processing, and device control based in bioelectric potentials. All related material is available on GitHub [12] from June 2016.

\section{ACKNOWLEDGMENT}

The authors would like to thank the Swedish Research Council (Vetenskapsrådet), VINNOVA, and Promobilia Foundation for funding this work.

\section{REFERENCES}

[1] P. Herberts, C. Almström, and K. Caine, "Clinical application study of multifunctional prosthetic hands", The Bone \& Joint Journal, vol. 60-B, no. 4, pp. 552-560, 1978.

[2] Schalk Lab, "BCI2000." [Online]. Available: http://www.schalklab.org/research/bci2000.

[3] "openEEG." [Online]. Available: http://openeeg.sourceforge.net/doc/index.html.

[4] openBCI, “openBCI." [Online]. Available: http://www.openbci.com/.

A. Beneteau, G. Di Caterina, L. Petropoulakis, and J. J. Soraghan, "Low-cost wireless surface EMG sensor using the MSP430 microcontroller", Proceeding for 6th European Embedded Design in Education and Research Conference, pp. 264-268, 2014.

[6] A. Ruvalcaba, A. Altamirano, C. Toledo, M. Ieee, R. Muñoz, and A. V. L. Leija, "Multichannel EMG Acquisition System for Arm and Forearm Signal Detection", Proceedings for IEEE International Instrumentation and Measuremement Technology Conference, pp. 1075 - 1078, 2014.

F. N. Jamaluddin, S. A. Ahmad, S. B. N. Noor, and W. Z. W. Hasan, "Low Cost and Wearable Multichannel Surface Electromyography Data Acquisition System Architecture", Journal of Enginnering Science and Technology, pp. 98-106, 2014 .

[8] M. Ortiz-Catalan, R. Brånemark, and B. Håkansson, "BioPatRec: A modular research platform for the control of artificial limbs based on pattern recognition algorithms", Source Code for Biology and Medicine, vol. 8, p. 11, 2013.

[9] E. Mastinu, M. Ortiz-catalan, and B. Håkansson, "Analog FrontEnds comparison in the way of a portable, low-power and lowcost EMG controller based on Pattern Recognition", Proceedings for IEEE Engineering in Medicine and Biology Society Conference, pp. 2111-2114, 2015.

[10] B. B. Winter and J. G. Webster, "Driven-right-leg circuit design", IEEE Transactions in Biomedical Engineering, vol. BME-30, no. 1, pp. 62-66, 1983.

[11] M. Ortiz-catalan, "BioPatRec." [Online]. Available: https://github.com/biopatrec/biopatrec/wiki.

[12] E. Mastinu and M. Ortiz-Catalan, "ADS BP." [Online]. Available: https://github.com/biopatrec/ADS_BP. 